\title{
Biological Therapeutic Advances for the Treatment of Advanced Urothelial Cancers
}

\author{
Mimma Rizzo (D) \\ Patrizia Giannatempo ${ }^{2}$ \\ Camillo Porta $\mathbb{D}^{1,3}$ \\ 'Division of Medical Oncology, A.O.U. \\ Consorziale Policlinico di Bari, Bari, Italy; \\ ${ }^{2}$ Department of Medical Oncology, \\ Fondazione I.R.C.C.S. Istituto Nazionale \\ dei Tumori, Milan, Italy; ${ }^{3}$ Department of \\ Biomedical Sciences and Human \\ Oncology, University of Bari 'A. Moro', \\ Bari, Italy
}

\begin{abstract}
In recent years, diagnostic and therapeutic advances have contributed to a reduction in mortality rates of patients with metastatic urothelial carcinoma (mUC). Immune checkpoint inhibitors have demonstrated efficacy and safety as both first-line and first-line switch maintenance therapy for mUC. For platinum-refractory patients, in addition to immunotherapy, other targeted agents (antibody-drug conjugates and fibroblast growth factor receptor inhibitors) have been approved after demonstrating a clinically relevant advantage in overall response rate, progression-free survival, and overall survival compared to standard of care. Sequential treatment strategies are finally feasible for patients with advanced urothelial carcinoma. This review will summarize the results of the most important phase II-III clinical trials for first-line, switch maintenance, second-line, and subsequent lines of therapy, and describe the most promising clinical trials currently ongoing in these treatment scenarios.
\end{abstract}

Keywords: metastatic urothelial carcinoma, targeted therapy, immune checkpoint inhibitors, first-line treatment, second-line treatment

\section{Introduction}

According to the GLOBOCAN 2020 estimates of cancer incidence and mortality, urothelial carcinoma (UC) is the tenth most commonly diagnosed cancer worldwide, being responsible for approximately 573,000 new cases and 213,000 deaths in $2020{ }^{1}$

As a whole, UC consists of both bladder cancer and upper urinary tract carcinomas arising from either the ureter or the renal pelvis. The main risk factors for these malignancies, which affect more males than females, are smoking, older age, family history of UC, infection with Schistosoma haematobium (in northern and sub-Saharan Africa), occupational exposure to aromatic amines and other chemicals affecting workers in the painting, rubber, or aluminum industries, and arsenic contamination of drinking water. ${ }^{2,3}$

As a direct consequence of both the average age and the frequent smoking habit of these patients, subjects with UC are often frail and highly comorbid, with renal impairment being highly prevalent among them. In terms of natural history, UC initially occurs as superficial, often multicentric, non-muscleinvasive, lesions, which are treated by means of repeated transurethral resections (often followed by intracavitary therapies); however, these lesions tend to recur repeatedly over the years until they become muscle invasive, leading to the need for radical cystectomy or nephroureterectomy (in the case of UC arising in the upper urinary tract), preceded or followed by systemic chemotherapy.,
Correspondence: Camillo Porta Department of Biomedical Sciences and Human Oncology, University of Bari "A. Moro", Piazza Giulio Cesare, II, Bari, 70124, Italy

Tel +39-080-5594167

Email camillo.porta@gmail.com 
Unfortunately, relapse after surgery occurs in approximately $30-50 \%$ of cases, often leading to the development of distant metastases; furthermore, $5 \%$ of all UC patients present with de novo metastatic disease. Both synchronous and metachronous metastatic urothelial carcinoma (mUC) patients have a very poor prognosis, although mortality rates have declined recently, at least in part as a consequence of therapeutic improvements, especially in more industrialized countries. Metastatic patients presenting with additional negative prognostic factors according to the Bajorin classification (ie low performance status and/or visceral metastases) have a particularly poor prognosis. ${ }^{6}$

In this review, we will summarize the most relevant phase II-III clinical trials conducted for first-line, maintenance of switch, second-line, and subsequent lines of therapy in advanced UC, and list ongoing trials.

\section{Cytotoxic Chemotherapy for Cisplatin-Eligible Metastatic UC Patients}

Locally advanced or metastatic (ie stage IV) UC has been shown to benefit from cytotoxic chemotherapy; despite therapeutic improvements made over the past few years, major international guidelines still recommend first-line cisplatin-containing combination chemotherapy for those patients who are fit enough to receive cisplatin., $5,7,8$

Among cisplatin-containing regimens, both the gemcitabine plus cisplatin doublet (GC) and the dose-dense MVAC (ddMVAC) polychemotherapy (which, together with cisplatin, also includes methotrexate, vinblastine, and doxorubicin), are commonly used as standard firstline treatments.

In 2000, a large, global, phase III study randomized 405 patients with locally advanced or metastatic UC to receive either GC or standard MVAC chemotherapy. At a median follow-up of 19 months, overall survival (OS) and time to progression (TTP) were similar in the two treatment arms; however, fewer toxic deaths were recorded among patients receiving GC compared with MVAC ( $1 \%$ vs $3 \%)$, a difference which was not statistically significant. A 5-year update analysis confirmed that GC was not superior to MVAC in terms of either OS (13.0\% vs $15.3 \%$, respectively) or progression-free survival (PFS) $\left(9.8 \%\right.$ vs $11.3 \%$, respectively). ${ }^{9}$

A subsequent large, randomized, phase III trial compared ddMVAC to standard MVAC. ${ }^{10}$ At a median follow- up of 7.3 years, $24.6 \%$ of ddMVAC-treated patients were alive, compared with $13.2 \%$ of those treated with standard MVAC; furthermore, less overall toxicity was reported in the ddMVAC group. ${ }^{11}$ Accordingly, standard MVAC was almost completely abandoned.

Despite the fact that GC had standard MVAC, and not ddMVAC, as the control arm of its pivotal trial, both GC and ddMVAC (with growth factor support) are the present standard of care for metastatic disease.

Although first-line platinum-containing chemotherapy results in objective responses or stable disease in approximately $65-75 \%$ of patients, it usually provides limited long-term benefits, in terms of both PFS (6-8 months) and OS (9-14 months).

In order to improve these results, taxane-based combinations were also tested; in particular, a randomized phase III trial was conducted to compare GC and GC plus paclitaxel in 626 patients with locally advanced or metastatic UC. The addition of paclitaxel to GC resulted in higher response rates and a borderline OS advantage, which was not statistically significant in the intention-totreat analysis; when eligible patients only were considered, the OS improvement for those patients treated with the three-drug regimen reached statistical significance (3.2 months), while no difference was recorded in terms of PFS. Furthermore, as expected, toxicity was worse for the three-drug combination, the incidence of neutropenic fever being $13.2 \%$ versus $4.3 \%$ for the doublet. ${ }^{12}$

Considering the limited benefit and the increase in severe toxicity observed, this combination ultimately did not replace the traditional standard of either GC or ddMVAC.

\section{Cytotoxic Chemotherapy for Cisplatin-Ineligible (or Unfit) Metastatic UC Patients}

Patients with renal impairment (estimated glomerular filtration rate $<50-60 \mathrm{~mL} / \mathrm{min}$ ), poor performance status (ECOG 2 or Karnofsky $\leq 70$ ), hearing loss (grade $\geq 2$ ), peripheral neuropathy (grade $\geq 2$ ), and/or symptomatic heart failure (NYHA class III/IV), who account for as many as $40 \%$ of all advanced UC patients, are considered cisplatin-ineligible or unfit; ${ }^{13}$ in this patient population, the doublet of carboplatin plus gemcitabine is the main recommended option. ${ }^{5,7,8}$

A phase II/III study assessed two carboplatincontaining regimens (carboplatin and gemcitabine or 
methotrexate, carboplatin, and vinblastine) in cisplatinunfit patients, mainly due to a performance status of 2; the overall response rate (ORR) was $42 \%$ and $30 \%$, respectively, but ORR dropped to $26 \%$ and $20 \%$, with an increased toxicity, among patients who had both a poor performance status and renal impairment. ${ }^{14}$

However, in the most recent phase III studies with combinations of immune checkpoint inhibitors (ICIs) and chemotherapy, regimens containing cisplatin and carboplatin demonstrated similar OS results to the historical benchmarks.

\section{Switch Maintenance Immunotherapy Following Cytotoxic Chemotherapy}

Since many chemotherapeutic agents, including platinum derivatives, may exert different immunogenic effects, the use of an ICI as a switch maintenance therapy following cytotoxic chemotherapy has been proposed.

Indeed, chemotherapy can induce immunogenic cell death, stimulate the antitumor immune responses through the release of "danger" signals from dying cells, ${ }^{15,16}$ deplete immunosuppressive populations (eg myeloidderived suppressor cells and regulatory $\mathrm{T}$ cells), ${ }^{17,18}$ stimulate tumor infiltration by $\mathrm{CD}^{+}$cytotoxic $\mathrm{T}$ lymphocytes, activate natural killer (NK) cells, enhance antigen presentation, stimulate the generation of tumor neoantigens, and also upregulate the expression of programmed death ligand-1 (PD-L1).

This concept was the basis of JAVELIN Bladder 100, a randomized, phase III trial aimed at evaluating the antiPD-L1 antibody avelumab as first-line switch maintenance therapy in 700 patients with stage IV UC. Enrolled patients had to have no progression after four to six cycles of first-line platinum-containing chemotherapy. After 4-10 weeks from the last dose of chemotherapy, patients were randomized to receive either avelumab (given at the dose of $10 \mathrm{mg} / \mathrm{kg}$, i.v., every 2 weeks) plus best supportive care (BSC) or BSC alone. Patients were also stratified by best response to first-line chemotherapy (complete or partial response [CR or PR] vs disease stabilization [SD]) and site of metastases (visceral vs non-visceral). The primary endpoint of the study was OS, assessed in two primary populations: all randomized patients and patients with PDL1-positive tumors, where PD-L1 positivity was evaluated by means of the Ventana SP263 assay, a subpopulation that accounted for $51 \%$ of the overall study group. The study succeeded, since avelumab plus BSC significantly prolonged OS vs BSC alone, median overall survival (mOS) being 21.4 months in the avelumab arm versus 14.3 months in the BSC-only arm, resulting in a hazard ratio (HR) of 0.69 (95\% confidence interval [CI] 0.56-0.86; $p=0.001$ ); that is, a $31 \%$ reduction in the risk of death. Avelumab also significantly prolonged OS in the PD-L1positive population; indeed, $\mathrm{mOS}$ was not reached in the avelumab arm versus 17.1 months in the control arm $(\mathrm{HR}=0.56,95 \%$ CI $0.40-0.79 ; p<0.001){ }^{19}$

An OS benefit with avelumab plus BSC versus BSC alone was observed across a number prespecified subgroups, including elderly ( $\geq 65$ years) patients $(\mathrm{HR}=0.63$, 95\% CI 0.47-0.83), patients with chronic kidney disease (creatinine clearance $[\mathrm{CrCl}]<60 \mathrm{~mL} /$ minute; $\mathrm{HR}=0.68$, 95\% CI 0.50-0.94), and patients with visceral, liver, or lung metastases $(\mathrm{HRs}=0.82,95 \%$ CI $0.62-1.09 ; 0.92,95 \%$ CI $\quad 0.54-1.56$; and $0.86,95 \%$ CI $0.56-1.30$, respectively). ${ }^{20}$ Furthermore, the OS benefit yielded by avelumab switch maintenance therapy was achieved despite the fact that, among patients progressing on study, $53 \%$ of those originally randomized to BSC alone (vs just $9 \%$ of those randomized to avelumab plus BSC) received a subsequent PD-1 or PD-L1 inhibitor. ${ }^{19,21}$

On the basis of the above results, avelumab was approved as a switch maintenance treatment for patients with locally advanced or metastatic UC whose disease had not progressed under first-line platinum-containing chemotherapy, and was thus granted the status of recommended treatment (level 1 evidence) in both the NCCN and ESMO guidelines. ${ }^{7,8}$

Although in the JAVELIN Bladder 100 trial avelumab was administered at the weight-based dose of $10 \mathrm{mg} / \mathrm{kg}$, the currently approved dose of avelumab, across different cancer types, is $800 \mathrm{mg}$ flat dose, every 2 weeks. This flat dosing was approved based on pharmacokinetic and pharmacodynamic analyses showing minimal differences compared with weight-based dosing across various weight ranges. ${ }^{22}$

In terms of safety, rates of grade $\geq 3$ treatment-related adverse events (TRAEs) were 17\% in avelumab-treated patients, with the most common grade $\geq 3$ TRAEs being increased lipase $(2.9 \%)$ and increased amylase $(2.0 \%){ }^{19}$ Furthermore, any grade and grade $\geq 3$ infusion reactions occurred in $10 \%$ and in $1 \%$ of avelumab-treated patients, respectively, despite the protocol requirement for premedication with an oral antihistamine and oral acetaminophen before the first four doses of the immunotherapeutic 
agent. As a whole, $12 \%$ and $10 \%$ of patients discontinued avelumab because of an adverse event of any cause and a TRAE, respectively. The rate of immune-related adverse events (irAEs) of any grade with avelumab was 29\%, including grade 3 events in $7 \%$, while $9 \%$ of all avelumabtreated patients needed high-dose corticosteroids $(\geq 40 \mathrm{mg}$ total daily prednisone or equivalent). ${ }^{19}$

Finally, median time to deterioration in the NCCN Functional Assessment of Cancer Therapy Bladder Cancer Symptom Index-18 (NFBISI-18) disease-related symptoms - physical subscale was similar between arms, clearly showing that avelumab had no detrimental effect on clinically relevant patient-reported outcomes. ${ }^{23}$

GU14-182 was a small, randomized, phase II trial aimed at comparing the anti-PD-L1 antibody pembrolizumab with placebo as first-line switch maintenance therapy in 107 patients with mUC who had not progressed under first-line platinum-containing chemotherapy. Pembrolizumab maintenance prolonged the primary endpoint of PFS (HR=0.65, 95\% CI not reported; log-rank $p=0.04)$, but not OS (HR=0.91, 95\% CI 0.52-1.59; $p=0.7477)$, which was a secondary endpoint. ${ }^{24}$

\section{Immune Checkpoint Inhibition for First-Line Treatment}

For the past three decades, first-line systemic therapy for patients with locally advanced or metastatic UC has consisted of platinum-doublet chemotherapy. Beyond chemotherapy, several antiangiogenic agents (bevacizumab, sunitinib, sorafenib) and targeted therapy have been tested in unselected patients, including inhibitors of epidermal growth factor receptor (EGFR, eg gefitinib and cetuximab) and human epidermal growth factor-2 (anti-HER-1-2, eg lapatinib and trastuzumab), which failed to demonstrate any activity.

In this landscape, the recent approval of monoclonal antibodies targeting programmed death ligand-1 (PD-L1) has revolutionized treatment, particularly in patients unfit for cisplatin-based chemotherapy (Table 1).

The initial proof of principle that ICIs show activity in patients with mUC was demonstrated in phase II trials (IMvigor210 with atezolizumab and KEYNOTE-052 with pembrolizumab) in cisplatin-unfit patients with advanced/metastatic UC. Since then, atezolizumab and pembrolizumab have quickly moved into two randomized phase III trials in the first-line setting. Following the results of these two trials, the role of immunotherapy has become a universal component of treatment in fragile patients in Europe (EMA) and the USA (FDA).

Accelerated FDA approval of atezolizumab and pembrolizumab for cisplatin-ineligible chemotherapy-naïve patients with advanced UC was based on single-arm studies (the IMvigor210 trial and the KEYNOTE-052 trial, respectively), which demonstrated favorable ORRs and duration of response (DoR). ${ }^{25,26}$

In the IMvigor210 trial, with a median follow-up period of 17 months, the ORR was 23\%, median PFS 2.7 months, mOS 15.9 months, 12-month survival rate 57\%, and mOS after stable disease 19.1 months; OS was considerably longer than the long-standing result obtained with gemcitabine/carboplatin $(9.3$ months $) .{ }^{27}$ In the KEYNOTE-052 trial, with a minimum follow-up of 24 months since the last patient enrolled, the ORR for patients with PD-L1-expressing and PD-L1-negative tumors was $47 \%$ and $20 \%$, median DoR was not reached, and mOS was 18.5 months and 9.7 months, respectively, suggesting PD-L1 as a powerful predictive biomarker for the response to pembrolizumab in the first-line setting. ${ }^{28}$

A subsequent large retrospective cohort study in a reallife cisplatin-ineligible population receiving pembrolizumab as first-line treatment confirmed lower short-term but superior long-term survival with ICIs and fewer adverse events compared to carboplatin-based chemotherapy. ${ }^{29}$

The combination of higher efficacy in terms of potential for durable benefit, the improvement in quality of life, and the favorable safety profile compared to carboplatinbased chemotherapy initially led the FDA and EMA to approve pembrolizumab and atezolizumab as standard first-line treatment in unselected cisplatin-unfit patients with mUC.

To enrich the response to immunotherapy, and based on the results of phase II studies, three randomized clinical trials were designed to evaluate the combination of ICIs and chemotherapy for previously untreated locally advanced or metastatic UC. Pembrolizumab, atezolizumab, and durvalumab were further evaluated in combination with chemotherapy, although the results and implications were less straightforward compared to phase II trial results.

The first trial was the IMvigor130 trial, which evaluated atezolizumab plus platinum-based chemotherapy, atezolizumab alone, and placebo plus platinum-based chemotherapy in 1213 patients. The median PFS of atezolizumab plus platinum-based chemotherapy was not statistically superior to platinum-based chemotherapy (8.2 vs 
Table I Phase II/III Trials of First-Line Immunotherapy

\begin{tabular}{|c|c|c|c|c|c|}
\hline Study (Phase) & Treatment (No. of Patients) & $\begin{array}{c}\text { mPFS } \\
\text { (Months) }\end{array}$ & $\begin{array}{c}\text { mOS } \\
\text { (Months) }\end{array}$ & $\begin{array}{c}\text { ORR } \\
\text { (\%) }\end{array}$ & $\begin{array}{c}\text { G3-4 } \\
\text { Treatment- } \\
\text { Related AEs }\end{array}$ \\
\hline \multicolumn{6}{|l|}{ Ist line-setting } \\
\hline \multirow[t]{3}{*}{ IMvigor2I0 (phase II) } & Atezolizumab (I19) & 2.7 & 15.9 & 23 & 16 \\
\hline & ICS $2 / 3$ & & 12.3 & 19 & \\
\hline & ICS $0 / 1$ & & 19.1 & 20 & \\
\hline \multirow[t]{3}{*}{ KEYNOTE-052 (phase II) } & Pembrolizumab (370) & & 11.3 & & 27 \\
\hline & CPS $<10$ & & 18.5 & 47 & \\
\hline & $C P S \geq 10$ & & 9.7 & 20 & \\
\hline \multirow[t]{5}{*}{ IMvigor I 30 (phase III) } & Atezolizumab + CDDP/CBDCA + gemcitabine $(45 \mathrm{I})$ & 8.2 & 16 & 47 & 85 \\
\hline & Atezolizumab (362) & \multirow[t]{2}{*}{6.3} & 15.7 & \multirow[t]{2}{*}{23} & \multirow[t]{2}{*}{42} \\
\hline & CPS $\geq 10$ & & 16.1 & & \\
\hline & CDDP/CBDCA + gemcitabine + placebo $(400)$ & 6.2 & 13.4 & 44 & 86 \\
\hline & CPS $\geq 10$ & & 15.2 & & \\
\hline \multirow[t]{3}{*}{ KEYNOTE-36I (phase III) } & Atezolizumab + CDDP/CBDCA + gemcitabine (35I) & 8.3 & 17.0 & 54.7 & 30 \\
\hline & Pembrolizumab (307) & & 15.6 & 30.3 & 1 \\
\hline & CDDP/CBDCA + gemcitabine + placebo (352) & 7.1 & 14.3 & 44.8 & 33 \\
\hline \multirow[t]{5}{*}{ DANUBE (phase III) } & Durvalumab + tremelimumab (342) & 3.7 & 15.1 & 36.2 & 27 \\
\hline & Durvalumab (346) & \multirow[t]{2}{*}{2.3} & & \multirow[t]{2}{*}{44.9} & \multirow[t]{2}{*}{14} \\
\hline & PD-LI positive & & 14.4 & & \\
\hline & CDDP/CBDCA + gemcitabine (344) & \multirow[t]{2}{*}{6.7} & 12.1 & \multirow[t]{2}{*}{49.1} & \multirow[t]{2}{*}{60} \\
\hline & PD-LI positive & & 12.1 & & \\
\hline
\end{tabular}

Abbreviations: mPFS, median progression-free survival; mOS, median overall survival; ORR, objective response rate; $A E$, adverse event; CDDP/CBDCA, cisplatin/ carboplatin; ICS, immune cell score; CPS, combined positive score; PD-LI, programmed death ligand-I.

6.3 months, respectively; $\mathrm{HR}=0.82,95 \%$ CI $0.70-0.96 ; p=$ 0.007). At a median follow-up of 11.8 months, mOS was 16 months for atezolizumab plus platinum-based chemotherapy and 13.4 months for platinum-based chemotherapy $\quad(\mathrm{HR}=0.83,95 \%$ CI $0.69-1.00$; one-sided $p=0.027)$. The ORR was $47 \%$ for atezolizumab plus platinum-based chemotherapy, $23 \%$ for atezolizumab alone, and $44 \%$ for platinum-based chemotherapy. ${ }^{30}$

Another phase III study of anti-PD-1 in combination with chemotherapy in first-line setting mUC was KEYNOTE-361, in which 1010 patients were randomly assigned to receive pembrolizumab in combination with platinum-based chemotherapy, pembrolizumab alone, or platinum-based chemotherapy. The prespecified dual primary endpoints of OS and PFS were not met, with a median progression-free survival (mPFS) in the pembrolizumab plus chemotherapy arm of 8.3 months compared with 7.1 months for chemotherapy alone (HR $=0.78,95 \%$ CI $0.65-0.93 ; p=0.003)$ and $\mathrm{mOS}$ of 17.0 versus 14.3 months, respectively ( $\mathrm{HR}=0.86,95 \% \mathrm{CI}$ $0.72-1.02 ; p=0.0407){ }^{31}$

In subgroup analyses of both studies, in patients with low PD-L1 expression the ICI arms had reduced survival compared to chemotherapy regimens. On the other hand, in patients with high PD-L1 expression there was a trend toward a better outcome in favor of patients treated with immunotherapy alone. In May 2018, based on these results, 
the FDA and EMA restricted the use of atezolizumab or pembrolizumab in the first-line setting to patients who are not eligible for cisplatin-containing chemotherapy and whose tumors express high PD-L1, as assessed by companion diagnostic tests, or patients who are considered unsuitable for any platinum-containing chemotherapy regardless of PD-L1 status.

A third study that should be mentioned in this therapeutic context is the DANUBE trial, which explored the role of durvalumab with or without tremelimumab versus platinumbased chemotherapy alone in 1032 patients with untreated, locally advanced, or metastatic UC. The co-primary endpoints were OS in patients with high PD-L1 expression treated with durvalumab as monotherapy compared to patients treated with chemotherapy, and OS in the intentionto-treat population treated with durvalumab plus tremelimumab compared to the population treated with chemotherapy. With a median follow-up of 41.2 months, the study did not meet any of its co-primary endpoints: in the population with high PD-L1 expression, OS was 14.4 months with durvalumab versus 12.1 months with chemotherapy $(\mathrm{HR}=0.89,95 \%$ CI $0.71-1.11 ; p=0.30$ ), while in the intention-to-treat population OS was 15.1 months with durvalumab plus tremelimumab versus 12.1 months (95\% CI 10.9-14.0) with chemotherapy $(\mathrm{HR}=0.85,95 \% \mathrm{CI} 0.72-1.02 ; p=0.075) .{ }^{32}$

Two additional ongoing studies are enrolling subjects to investigate the role of combination immunotherapy plus chemotherapy in this population. The NILE trial (NCT03682068) is exploring chemotherapy with or without durvalumab alone or in combination with tremelimumab, and the CheckMate 901 trial (NCT03036098) is comparing the combination of nivolumab and ipilimumab versus chemotherapy in patients ineligible for cisplatin and in patients with elevated PD-L1, along with a substudy evaluating standard cisplatin-based chemotherapy with or without nivolumab. Furthermore, the LEAP-011 trial (NCT03898180) is evaluating pembrolizumab in combination with lenvatinib versus pembrolizumab plus placebo for first-line treatment of cisplatin-ineligible patients with a combined positive score (CPS) $\geq 10$ and platinumineligible patients.

\section{Immune Checkpoint Inhibitors and Targeted Therapies for Second-Line Treatment}

Seventy percent of patients receiving platinum-based chemotherapy develop rapid progression. Several salvage therapies are currently approved for patients who have progressed to platinum-based chemotherapy, including checkpoint inhibitors (Table 2), fibroblast growth factor receptor (FGFR) inhibitors, and antiangiogenic agents.

Among PD-L1 checkpoint inhibitors, only pembrolizumab, in a randomized phase III trial, KEYNOTE-045, showed a survival benefit (OS 10.3 vs 7.4 months, $p=0.002$ ) versus chemotherapy with paclitaxel, docetaxel, or vinflunine, although there was no difference in PFS. The ORR for the pembrolizumab group was higher than for the chemotherapy group $(21.1 \%$ vs $11.4 \%, p=0.001)$ and comparable between PD-L1 low and PD-L1 high expression subgroups. Treatment-related serious adverse events were reported in $15 \%$ of patients receiving pembrolizumab; the treatment discontinuation rate was $5.6 \%{ }^{33}$

Different results were seen in the phase III study IMvigor211, which showed no benefit in OS or PFS for atezolizumab compared to chemotherapy. Treatmentrelated serious adverse events were reported in $6 \%$ of patients receiving pembrolizumab; the treatment discontinuation rate was $3 \%^{34}$

In addition, the results of the SAUL trial have recently been published. This was the first prospective single-arm study to confirm the safety and efficacy of atezolizumab in a large, real-world pretreated population with urinary tract carcinoma, including patients with symptomatic brain metastasis, autoimmune disease, ECOG $>1$, inadequate kidney function, or non-urothelial histology. The mOS was 8.7 months (95\% CI 7.8-9.9), mPFS was 2.2 months (95\% CI 2.1-2.4), and the ORR was $13 \%$ (95\% CI 11$16 \% ; 3 \% \mathrm{CR}) .{ }^{35}$

Of note, contradictory results have been reported in these two large trials on the use of PD-L1 expression as biomarker-driven patient selection. The atezolizumab trial reported a lack of consistency on OS data in PD-L1positive patients; in contrast, the pembrolizumab trial showed a relevant improved outcome in patients with high expression of PD-L1 (18.5 vs 9.7 months). Direct comparisons should not be made owing to the different definition of PD-L1 positivity in these two trials; thus, any conclusions are highly speculative and uncertain.

Nivolumab, durvalumab, and avelumab currently have no OS data in prospective randomized phase III trials, although in phase II trials similar proportions of patients (17-20\%) achieve an overall response.

Another intriguing approach is to explore the molecularly targeted therapies in selected patients with UC. In particular, the dysregulation of fibroblast growth factor 
Table 2 Phase II/III Trials of Second-Line Targeted Therapy

\begin{tabular}{|c|c|c|c|c|c|}
\hline Study (Phase) & Treatment (No. of Patients) & $\begin{array}{c}\text { mPFS } \\
\text { (Months) }\end{array}$ & $\begin{array}{c}\text { mOS } \\
\text { (Months) }\end{array}$ & $\begin{array}{c}\text { ORR } \\
\text { (\%) }\end{array}$ & $\begin{array}{c}\text { G3-4 } \\
\text { Treatment- } \\
\text { Related AEs }\end{array}$ \\
\hline \multicolumn{6}{|l|}{ Second-line setting } \\
\hline \multirow[t]{2}{*}{ KEYNOTE-045 (phase III) } & Pembrolizumab (270) & 2.1 & 10.3 & 21.1 & 15 \\
\hline & Paclitaxel/docetaxel/vinflunine (272) & 3.3 & 7.4 & 11.4 & 49.4 \\
\hline \multirow[t]{8}{*}{ IMvigor2II (phase III) } & Atezolizumab (459) & \multirow[t]{4}{*}{2.1} & 8.6 & \multirow[t]{4}{*}{13.2} & \multirow[t]{4}{*}{53} \\
\hline & $\mathrm{ICl} 2 / 3$ & & II.I & & \\
\hline & $\mathrm{ICl} \mathrm{I/2/3}$ & & 8.9 & & \\
\hline & $\mathrm{ICl} 0$ & & 7.2 & & \\
\hline & Paclitaxel/docetaxel/vinflunine (443) & & 8.0 & \multirow[t]{4}{*}{13.3} & \multirow[t]{4}{*}{56} \\
\hline & $\mathrm{ICl} 2 / 3$ & & 10.6 & & \\
\hline & $\mathrm{ICl} \mathrm{I/2/3}$ & & 8.2 & & \\
\hline & $\mathrm{ICl} 0$ & & 6.7 & & \\
\hline SAUL (phase II) & Atezolizumab (696) & 2.2 & 8.7 & 13 & 41 \\
\hline Erdafitinib (phase II) & Erdafitinib (99) & 5.5 & 13.8 & 40 & 67 \\
\hline \multirow[t]{2}{*}{ RANGE trial (phase III) } & Ramucirumab + docetaxel (263) & 4.1 & 9.4 & 25.9 & 44.5 \\
\hline & Docetaxel (267) & 2.8 & 7.9 & 13.9 & 38.8 \\
\hline
\end{tabular}

Abbreviations: mPFS, median progression-free survival; mOS, median overall survival; ORR, objective response rate; $A E$, adverse event; ICS, immune cell score.

signaling (FGF) can occur in around $20 \%$ of patients with mUC via $F G F R$ gene alteration, such as mutation, fusion, or amplification. ${ }^{36}$ The fibroblast growth factor-3 gene (FGFR3) has long been associated with bladder oncogenesis, and FGFR 3 inhibition is becoming an interesting treatment approach for selected patients.

In April 2019, the US FDA approved erdafitinib for locally advanced or metastatic platinum-refractory UC with at least one FGFR3 alteration or FGFR2/3-containing fusion. BLC2001, an open-label phase II clinical trial, enrolled 99 patients with prespecified $F G F R 2 / 3$ alterations which progressed during or following at least one line of prior chemotherapy for locally advanced UC or unresectable/ metastatic UC. After a median follow-up of 11.2 months, $40 \%$ of patients had an objective response to erdafitinib. A higher ORR (59\%) was observed among patients who had previously received ICIs. With a median follow-up of 24 months, the mPFS was 5.52 months (95\% CI 4.2-6.0) and the mOS was 11.3 months ( $95 \%$ CI 9.8 to not reached). ${ }^{37}$

Other FGFR inhibitors, including rogaratinib, infigratinib, and pemigatinib, were tested in phase I and II studies and showed an ORR of around $25 \%$. The results of a phase II study of vofatamab, a selective inhibitor of FGFR3, as salvage therapy in $\mathrm{mUC}$, have recently been presented, with an ORR of $10 \%$ in monotherapy and $33 \%$ in combination with docetaxel. In the phase I/II FIERCE-22 study, the combination of vofatamab and pembrolizumab in previously pretreated mUC with and without FGFR3 alterations resulted in an ORR of $33 \%$ in wild-type tumors and $43 \%$ in tumors with FGFR3 alterations. Phase $\mathrm{Ib} / \mathrm{II}$ trials with combinations of erdafitinib/certrelimab (NCT03473743), pemigatinib/pembrolizumab (NCT04003610), and rogaratinib/atezolizumab (NCT03473756) are currently ongoing.

In addition, in this landscape even angiogenic agents have been investigated in UC, based on encouraging results of phase II trials; however, the two phase III trials did not confirm the preliminary results when used alone or in combination with chemotherapy.

The phase III trial CALGB90601 compared bevacizumab, a monoclonal antibody against vascular endothelial growth factor-A (VEGF-A), plus chemotherapy (gemcitabine and cisplatin, GC) versus placebo plus chemotherapy as first-line therapy. The addition of bevacizumab to chemotherapy did not result in improved OS (14.5 vs 14.3 months; $\mathrm{HR}=0.87,95 \%$ 
CI 0.72-1.05; two-sided stratified log-rank $p=0.14) .{ }^{38}$ The phase III RANGE evaluated ramucirumab, a monoclonal antibody to VEGFR-2, plus docetaxel versus placebo plus docetaxel in patients with locally advanced or metastatic UC who progressed on platinum-based chemotherapy. Ramucirumab plus docetaxel improved PFS (4.1 months, 95\% CI 3.3-4.8, vs 2.8 months, 95\% CI 2.6-2.9; HR $=0.696$, 95\% CI $0.573-$ $0.845 ; p=0.0002$ ), without a significant improvement in OS (9.4 months; 95\% CI 7.9-11.4, in the ramucirumab group versus 7.9 months, 95\% CI 7.0-9.3, in the placebo group; stratified $\mathrm{HR}=0.887$, 95\% CI $0.724-1.086 ; p=0.25$ ), for patients with platinum-refractory advanced UC. ${ }^{39}$

In conclusion, although the combinations of antiVEGFR and chemotherapy showed a prolonged PFS, the inhibition of VEGFR signaling was not effective in improving OS in patients with mUC.

\section{Beyond Second-Line Treatment}

Despite the recent advances, both chemotherapy and immunotherapy have had limited success, with long-term response rates of $20-30 \%$ in patients with mUC. Up to $70-80 \%$ of patients experience disease progression or relapse after chemotherapy and immunotherapy. Therefore, there is an urgent need for effective new agents to overcome resistance to cancer treatment and delay the relapse of the tumor. Antibody-drug conjugate (ADC) agents are a promising new therapeutic option that utilizes antibody-mediated delivery of a potent cytotoxic payload directly to the cancer cell, and represents a shift in the treatment of advanced UC (Table 3).

The first ADC drug approved by the FDA was enfortumab vedotinib (EV), a humanized monoclonal antibody linked to the chemotoxic microtubule-disrupting agent monomethyl auristatin E (MMAE), which targets nectin4, a transmembrane protein widely expressed and associated with poor prognosis in mUC tumors.

After the phase I/II study yielded promising results, the phase III study EV-301 compared EV to investigator-chosen chemotherapy (docetaxel, paclitaxel, or vinflunine) in patients with locally advanced or metastatic UC who had previously been treated with ICI and platinum-based chemotherapy. This trial was stopped early owing to positive interim analysis. The results demonstrated a significant improvement in mOS, which was 12.88 months (95\% CI 8.05-10.74) with EV versus 8.97 months (95\% CI 8.05-10.74) with chemotherapy; mPFS was 5.55 months (95\% CI 5.32-5.82) with EV versus 3.71 months (95\% CI 3.52-3.94) with chemotherapy $(\mathrm{HR}=0.62$, $95 \%$ CI $0.51-0.75 ; p<0.001$ ); ORR was $40.6 \%$ with EV versus $17.9 \%$ with chemotherapy ( $p<0.001)$; and the median DoR to EV was 7.39 months. ${ }^{40}$ The most common side effects were rash, peripheral neuropathy, fatigue, and alopecia. It is important to note the ocular toxicity and hyperglycemia.

These promising data and preliminary results of synergic effects when combined with immunotherapy have led to research evaluating the efficacy of EV in other earlier treatment scenarios, with two studies currently underway: the EV302 study is evaluating EV in combination with pembrolizumab in the first-line setting and the KEYNOTE-915 study is evaluating the same combination in the perioperative setting.

Other exciting ADCs are currently under development are sacituzumab govitecan (a conjugate formed by an antibody against trop 2 and SN-38 and an active metabolite of irinotecan), RC48-ADC (a conjugate formed by an antibody against HER-2 and MMAE), and trastuzumab deruxtecan (a conjugate formed by an antibody against HER-2 and a topoisomerase-1 inhibitor). The response rates observed with these ADCs in phase I trials are extremely promising.

Intriguing new results have been reported from phase I/ II data on sacituzumab, which showed an ORR of $31 \%$ in 45 patients with advanced UC. ${ }^{41}$ A phase II study (NCT03547973) is currently ongoing.

\section{Conclusions}

The therapeutic landscape of advanced urothelial carcinoma has been enriched with additional classes of agents.

Table 3 Phase II/III Trials with Targeted Therapies Beyond Second-Line Treatment

\begin{tabular}{|l|l|l|l|l|l|}
\hline Study (Phase) & \multicolumn{1}{|c|}{ Treatment (No. of Patients) } & \multicolumn{1}{|c|}{$\begin{array}{c}\text { mPFS } \\
\text { (Months) }\end{array}$} & \multicolumn{1}{|c|}{$\begin{array}{c}\text { mOS } \\
\text { (Months) }\end{array}$} & $\begin{array}{c}\text { ORR } \\
\text { (\%) }\end{array}$ & $\begin{array}{c}\text { G3-4 Treatment- } \\
\text { Related AEs }\end{array}$ \\
\hline Beyond second line & \multicolumn{5}{|l|}{} \\
\hline EV-30I (phase III) & Enfortumab vedotinib (30I) & 5.55 & 12.88 & 40.6 & 51.4 \\
\hline & Docetaxel/paclitaxel/vinflunine (30I) & 3.71 & 8.97 & 17.9 & 49.8 \\
\hline THROPY-U-0I (phase II) & Sacituzumab (II3) & 5.4 & 10.9 & 27 & \\
\hline
\end{tabular}

Abbreviations: mPFS, median progression-free survival; mOS, median overall survival; ORR, objective response rate; $A E$, adverse event. 
Cisplatin-based chemotherapy remains the first-line standard treatment for cisplatin-eligible patients. For cisplatin-ineligible patients, ICIs can be used in PD-L1-high patients.

For second-line treatment, among the FDA-approved ICIs, pembrolizumab is the only one with an OS benefit observed in a randomized phase III trial (KEYNOTE-045).

The ORR of targeted therapies is significantly higher than for cytotoxic drugs in patients refractory to platinumbased chemotherapy.

For patients with actionable FGFR2 or FGFR3 genomic alterations, erdafitinib is the first-choice treatment option after platinum-based treatment.

The results (benefit in OS, PFS, and ORR) obtained with EV in patients who failed both platinum and ICI have never been achieved before.

Since the therapeutic armamentarium for advanced urothelial carcinoma is finally being enriched, we need to identify the clinical and molecular factors that can help to select the optimal treatment sequence for a specific patient.

\section{Funding}

The authors have no funding or financial support to disclose.

\section{Disclosure}

MR acted as a paid consultant and/or speaker for Pfizer, Novartis, MSD and AstraZeneca. PG acted as a paid consultant and/or speaker for Astellas Pharma, Janssen and MSD, and received research funding from MSD, Astra Zeneca and Ipsen, and travel accommodation from Janssen. CP acted as a paid consultant and/or speaker for Angelini Pharma, AstraZeneca, BMS, Eisai, EUSA Pharma, General Electric, Ipsen, Janssen, Merck, MSD, Novartis and Pfizer, as an expert testimony for EUSA Pharma and Pfizer, as a Protocol Steering Committee Member for BMS, Eisai and EUSA Pharma, and received travel accommodation from Roche. The authors report no other conflicts of interest in this work.

\section{References}

1. Sung H, Ferlay J, Siegel RL, et al. Global cancer statistics 2020: GLOBOCAN estimates of incidence and mortality worldwide for 36 cancers in 185 countries. CA Cancer J Clin. 2021;71(3):209-249. doi: $10.3322 /$ caac. 21660

2. Antoni S, Ferlay J, Soerjomataram I, et al. Bladder cancer incidence and mortality: a global overview and recent trends. Eur Urol. 2017;71 (1):96-108. doi:10.1016/j.eururo.2016.06.010

3. Richters A, Aben KKH, Kiemeney L. The global burden of urinary bladder cancer: an update. World J Urol. 2020;38:1895-1904. doi:10.1007/s00345-019-02984-4
4. Babjuk M, Burger M, Compérat EM, et al. European Association of Urology guidelines on non-muscle-invasive bladder cancer (TaT1 and carcinoma in situ) - 2019 update. Eur Urol. 2019;76 (5):639-657. doi:10.1016/j.eururo.2019.08.016

5. Witjes JA, Bruins HM, Cathomas R, et al. European Association of Urology guidelines on muscle-invasive and metastatic bladder cancer: summary of the 2020 guidelines. Eur Urol. 2021;79(1):82-104. doi:10.1016/j.eururo.2020.03.055

6. Bajorin DF, Dodd PM, Mazumdar M, et al. Long-term survival in metastatic transitional-cell carcinoma and prognostic factors predicting outcome of therapy. J Clin Oncol. 1999;17(10):3173-3181. doi:10.1200/JCO.1999.17.10.3173

7. Bellmunt Orsola JA, Leow JJ, Wiegel T, et al. The bladder cancer: ESMO clinical practice guidelines for diagnosis, treatment and follow-up. Ann Oncol. 2014;25(Suppl 3):iii40-iii48. doi:10.1093/ annonc/mdu223

8. Fleig TW, Spiess PE, Agarwal N, et al. Bladder cancer, version 3.2020, NCCN clinical practice guidelines in oncology. $J$ Natl Compr Canc Netw. 2020;18:329-354. doi:10.6004/jnccn.2020.0011

9. von der Maase H, Hansen SW, Roberts JT, et al. Gemcitabine and cisplatin versus methotrexate, vinblastine, doxorubicin, and cisplatin in advanced or metastatic bladder cancer: results of a large, randomized, multinational, multicenter, phase III study. J Clin Oncol. 2000;18:3068-3077. doi:10.1200/JCO.2000.18.17.3068

10. Sternberg CN, de Mulder PH, Schornagel JH, et al. Randomized phase III trial of high-dose-intensity methotrexate, vinblastine, doxorubicin, and cisplatin (MVAC) chemotherapy and recombinant human granulocyte colony-stimulating factor versus classic MVAC in advanced urothelial tract tumors: European Organization for research and treatment of cancer protocol no. 30924. J Clin Oncol. 2001;19:2638-2646.

11. Sternberg CN, de Mulder P, Schornagel JH, et al. Seven-year update of an EORTC phase III trial of high-dose intensity M-VAC chemotherapy and G-CSF versus classic M-VAC in advanced urothelial tract tumours. Eur J Cancer. 2006;42:50-54. doi:10.1016/j.ejca.2005.08.032

12. Bellmunt J, von der Maase H, Mead GM, et al. Randomized phase III study comparing paclitaxel/cisplatin/gemcitabine and gemcitabine/ cisplatin in patients with locally advanced or metastatic urothelial cancer without prior systemic therapy: EORTC Intergroup Study 30987. J Clin Oncol. 2012;30:1107-1113. doi:10.1200/JCO.2011.38.6979

13. Galsky MD, Hahn NM, Rosenberg J. Treatment of patients with metastatic urothelial cancer "unfit" for cisplatin-based chemotherapy. J Clin Oncol. 2011;29:2432-2438. doi:10.1200/JCO.2011.34.8433

14. De Santis M, Bellmunt J, Mead G, et al. Randomized phase II/III trial assessing gemcitabine/ carboplatin and methotrexate/carboplatin/vinblastine in patients with advanced urothelial cancer "unfit" for cisplatin-based chemotherapy: phase II results of EORTC study 30986. J Clin Oncol. 2009;27:5634-5639. doi:10.1200/JCO.2008.21.4924

15. de Biasi AR, Villena-Vargas J, Adusumilli PS. Cisplatin-induced antitumor immunomodulation: a review of preclinical and clinical evidence. Clin Cancer Res. 2014;20:5384-5391. doi:10.1158/10780432.CCR-14-1298

16. Galluzzi L, Buque A, Kepp O, et al. Immunological effects of conventional chemotherapy and targeted anticancer agents. Cancer Cell. 2015;28(6):690-714. doi:10.1016/j.ccell.2015.10.012

17. Chen C, Chen Z, Chen D, Zhang B, Wang Z, Le H. Suppressive effects of gemcitabine plus cisplatin chemotherapy on regulatory $\mathrm{T}$ cells in nonsmall-cell lung cancer. $J$ Int Med Res. 2015;43 (2):180-187. doi:10.1177/0300060514561504

18. Eriksson E, Wenthe J, Irenaeus $\mathrm{S}$, et al. Gemcitabine reduces MDSCs, tregs and TGF $\beta-1$ while restoring the teff/treg ratio in patients with pancreatic cancer. J Transl Med. 2016;14:282. doi:10.1186/s12967-016-1037-z

19. Powles T, Park SH, Voog E, et al. Avelumab maintenance therapy for advanced or metastatic urothelial carcinoma. $N$ Engl J Med. 2020;383 (13):1218-1230. doi:10.1056/NEJMoa2002788 
20. Grivas P, Park SH, Voog E, et al. 704MO avelumab first-line (1L) maintenance + best supportive care (BSC) vs BSC alone with $1 \mathrm{~L}$ chemotherapy (CTx) for advanced urothelial carcinoma (UC): subgroup analyses from JAVELIN bladder 100. Ann Oncol. 2020;31: S550(abs. 704MO). doi:10.1016/j.annonc.2020.08.776

21. Powles T, Park SH, Voog E, et al. Maintenance avelumab + best supportive care (BSC) versus BSC alone after platinum-based first-line $(1 \mathrm{~L})$ chemotherapy in advanced urothelial carcinoma (UC): JAVELIN bladder 100 phase III interim analysis. $J$ Clin Oncol. 2020;38:abs.LBA1. doi:10.1200/JCO.2020.38.18_suppl. LBA1

22. Novakovic AM, Wilkins JJ, Dai $\mathrm{H}$, et al. Changing body weight-based dosing to a flat dose for avelumab in metastatic Merkel cell and advanced urothelial carcinoma. Clin Pharmacol Ther. 2020;107:588-596. doi:10.1002/cpt.1645

23. Powles T, Kopyltsov E, Su P-J, et al. Patient-reported outcomes (PROs) from JAVELIN bladder 100: avelumab first-line (1L) maintenance + best supportive care (BSC) vs BSC alone for advanced urothelial carcinoma (UC). Ann Oncol. 2020;31:S550(Abs. 745P).

24. Galsky MD, Mortazavi A, Milowsky MI, et al. Randomized double-blind phase II study of maintenance pembrolizumab versus placebo after first-line chemotherapy in patients with metastatic urothelial cancer. $J$ Clin Oncol. 2020;38(16):1797-1806. doi:10.1200/JCO.19.03091

25. Balar AV, Galsky MD, Rosenberg JE, et al. Atezolizumab as first-line treatment in cisplatin-ineligible patients with locally advanced and metastatic urothelial carcinoma: a single-arm, multicentre, Phase 2 trial. Lancet. 2017;389(10064):67-76. doi:10.1016/S0140-6736(16) 32455-2

26. Balar AV, Castellano D, O'Donnell PH, et al. First-line pembrolizumab in cisplatin-ineligible patients with locally advanced and unresectable or metastatic urothelial cancer (KEYNOTE-052): a multicentre, single-arm, phase 2 study. Lancet Oncol. 2017;18 (11):1483-1492. doi:10.1016/S1470-2045(17)30616-2

27. De Santis M, Bellmunt J, Mead G, et al. Randomized phase II/III trial assessing gemcitabine/carboplatin and methotrexate/carboplatin/vinblastine in patients with advanced urothelial cancer who are unfit for cisplatin- based chemotherapy: EORTC study 30986. J Clin Oncol. 2012;30:191-199. doi:10.1200/JCO.2011.37.3571

28. Vuky J, Balar AV, Castellano D, et al. Long-term outcomes in KEYNOTE-052: phase II study investigating first-line pembrolizumab in cisplatin-ineligible patients with locally advanced or metastatic urothelial cancer. J Clin Oncol. 2020;38(23):2658-2666. doi:10.1200/JCO.19.01213

29. Feld E, Harton J, Meropol NJ, et al. Effectiveness of first- line immune checkpoint blockade versus carboplatin- based chemotherapy for metastatic urothelial cancer. Eur Urol. 2019;76:524-532. doi:10.1016/j.eururo.2019.07.032

30. Galsky MD, Arija JAA, Bamias A, et al. Atezolizumab with or without chemotherapy in metastatic urothelial cancer (IMvigor130): a multicentre, randomised, placebo-controlled phase 3 trial. Lancet. 2020;395:1547-1557. doi:10.1016/S0140-6736(20)30230-0
31. Alva A, Csõszi T, Ozguroglu M, et al. LBA23 pembrolizumab (P) combined with chemotherapy (C) vs $\mathrm{C}$ alone as first-line (1L) therapy for advanced urothelial carcinoma (UC): KEYNOTE-361. Ann Oncol. 2020;31(suppl_4):S1142-S1215. doi:10.1016/j.annonc.20 20.08.2252

32. Powles TB, van der Heijden MS, Castellano Gauna D. A Phase III, randomized, open-label study of first-line durvalumab (D) with or without tremelimumab (T) vs standard of care chemotherapy in patients with unresectable, locally advanced or metastatic urothelial carcinoma (DANUBE). Ann Oncol. 2020;31(suppl_4):S550. doi:10.1016/j.annonc.2020.08.769

33. Bellmunt J, De Wit R, Vaughn DJ, et al. Pembrolizumab as second-line therapy for advanced urothelial carcinoma. $N$ Engl $J$ Med. 2017;376:1015-1026. doi:10.1056/NEJMoa1613683

34. Powles T, Durán I, van der Heijden MS, et al. Atezolizumab versus chemotherapy in patients with platinum-treated locally advanced or metastatic urothelial carcinoma (IMvigor211): a multicentre, open-label, phase 3 randomised controlled trial. Lancet. 2018;391 (10122):690-714. doi:10.1016/S0140-6736(17)33297-X

35. Sternberg CN, Loriot Y, James N, et al. Primary results from SAUL, a multinational single-arm safety study of atezolizumab therapy for locally advanced or metastatic urothelial or nonurothelial carcinoma of the urinary tract. Eur Urol. 2019;76(1):73-81. doi:10.1016/j. eururo.2019.03.015

36. Necchi A, Lo Vullo S, Raggi D, et al. Prognostic effect of FGFR mutations or gene fusions in patients with metastatic urothelial carcinoma receiving first-line platinum-based chemotherapy: results from a large, single-institution cohort. Eur Urol Focus. 2019;5 (5):853-856. doi:10.1016/j.euf.2018.02.013

37. Loriot Y, Necchi A, Park SH, et al. Erdafitinib in locally advanced or metastatic urothelial carcinoma. $N$ Engl $J$ Med. 2019;381 (4):338-348. doi:10.1056/NEJMoa1817323

38. Rosenberg JE, Ballman KA, Halabi S, et al. Randomized Phase III trial of gemcitabine and cisplatin with bevacizumab or placebo in patients with advanced urothelial carcinoma: results of CALGB 90601 (Alliance). J Clin Oncol. 2021;2486-2496. doi:10.1200/ JCO.21.00286

39. Petrylak DP, de Wit R, Chi KN, et al. Ramucirumab plus docetaxel versus placebo plus docetaxel in patients with locally advanced or metastatic urothelial carcinoma after platinum-based therapy (RANGE): overall survival and updated results of a randomised, double-blind, phase 3 trial. Lancet Oncol. 2020;21(1):105-120. doi:10.1016/S1470-2045(19)30668-0

40. Powles T, Rosenberg JE, Sonpavde GP, et al. Enfortumab vedotin in previously treated advanced urothelial carcinoma. $N$ Engl J Med. 2021;384(12):1125-1135. doi:10.1056/NEJMoa2035807

41. Tagawa ST, Faltas BM, Lam ET, et al. Sacituzumab govitecan (IMMU-132) in patients with previously treated metastatic urothelial cancer (mUC): results from a phase I/II study. J Clin Oncol. 2019;37 (7_suppl):354. doi:10.1200/JCO.2019.37.7_suppl.354

\section{Publish your work in this journal}

Biologics: Targets and Therapy is an international, peer-reviewed journal focusing on the patho-physiological rationale for and clinical application of Biologic agents in the management of autoimmune diseases, cancers or other pathologies where a molecular target can be identified. This journal is indexed on PubMed Central, CAS, EMBase,
Scopus and the Elsevier Bibliographic databases. The manuscript management system is completely online and includes a very quick and fair peer-review system, which is all easy to use. Visit http://www.dovepress.com/testimonials.php to read real quotes from published authors. 